\title{
Deep sequencing analysis of the circadian transcriptome of the jewel wasp Nasonia vitripennis
}

\author{
Nathaniel J. Davies and Eran Tauber ${ }^{\dagger}$
}

Dept. of Genetics, University of Leicester, University Road, Leicester LE1 7RH, UK

Running title: Sequencing the Nasonia circadian transcriptome

Keywords: Circadian clock; transcriptomics; Nasonia; RNAseq

\footnotetext{
${ }^{\dagger}$ For correspondence. Dept. Genetics, University of Leicester, University Road, Leicester LE1 7RH, United Kingdom. Email: et22@le.ac.uk

Tel: +44-116- 252- 3455; Fax; +44 116252378
}

1 


\section{Abstract}

3 The study of the circadian clock has benefited greatly from using Drosophila as a

4 model system. Yet, accumulating evidence suggests that the fly might not be the

5 canonical insect model. Here, we have analysed the circadian transcriptome of

6 the Jewl wasp Nasonia vitripennis by using RNA-seq in both constant darkness

7 and constant light (in contrast to flies, the wasps are rhythmic under continuous

8 light). We identify approximately $6 \%$ of the transcriptome as cycling under

9 constant conditions, revealing a bimodal distribution of phases and low cycling

10 amplitude. We examine the functions under circadian control in Nasonia,

11 identifying clock control of functions such as metabolism, light response, and a

12 variety of neural processes, drawing comparisons between Nasonia and

13 Drosophila. We characterise the transcriptional differences underlying

14 phenotypic differences in free-running circadian behaviour in constant darkness

15 and constant light, revealing significant down-regulation of catabolic processes

16 in constant light. We also profile levels of opsins transcription, gaining insight

17 into how Nasonia responds to light, which is a key question in circadian research

18 in the Hymenoptera. Although there was little similarity between cycling genes

19 in Drosophila and Nasonia, the functions fulfilled by cycling transcripts were

20 similar in both species. Of the known Drosophila core clock genes, only $p d p 1 e$,

21 shaggy and Clock showed a significant cycling in Nasonia, underscoring the

22 importance of studying the clock in non-model organisms. 
25 The circadian clock regulates fundamental biological processes such as sleep

26 (Huang, et al. 2011), metabolism (Huang, et al. 2011), and the immune system

27 (Scheiermann, et al. 2013), and has implications for a wide range of human

28 diseases. Notable examples of diseases linked to the circadian clock include

29 cancer (Kelleher, et al. 2014), Alzheimer's disease (Musiek, et al. 2015),

30 cardiovascular disease (Takeda and Maemura, 2011), obesity (Maury, et al.

31 2010), diabetes (Maury, et al. 2010), and depression (Quera Salva, et al. 2011). A

32 primary output of the clock is circadian regulation of transcription, a trait which

33 has been demonstrated in mammals (Hughes, et al. 2009), insects (McDonald

34 and Rosbash, 2001a), plants (Schaffer, et al. 2001), and even bacteria (Woelfle

35 and Johnson, 2006). Therefore, analysing transcriptional oscillations in clock-

36 controlled genes (CCGs) is a key step in understanding how the daily rhythms

37 produced by the clock are ultimately linked to behavioural phenotypes.

38 The genetic mechanisms underlying the animal circadian clock were first

39 elucidated through studies of model animals; primarily the fruit fly Drosophila.

40 The first clock gene to be identified, period (per), was discovered through

41 mapping the genetic basis of Drosophila mutants with aberrant locomotor and

42 eclosion rhythms (Konopka and Benzer, 1971). The discovery of period was

43 followed by the discovery of its heterodimeric partner timeless (tim) (Sehgal, et

44 al. 1994). These two genes are joined by a roster of other genes working together

45 to produce robust internal rhythms.

46 The discoveries made in Drosophila have been instrumental for

47 understanding the mechanisms of the circadian clock in mammals (Yu and

48 Hardin, 2006). As the principal insect model, Drosophila has been used to great 
effect to model circadian phenomena in humans (Rosato, et al. 2006). However, as circadian research into non-drosophilid insects has advanced, several alternative clock models have been proposed (Yuan, et al. 2007), some of which may better model aspects of the mammalian clock than Drosophila.

For example, a major difference between the various clock models in insects concerns the light input pathway. The main light input to the clock in Drosophila is mediated through cryptochrome (cry1) which is activated in response to light (Ceriani, et al. 1999), binds to and promotes the degradation of tim (Busza, et al. 2004), ultimately resulting in the degradation of per (Ko, et al. 2002,Grima, et al. 2002). In contrast, mammalian-like cryptochrome (cry2) is not light-sensitive (Yuan, et al. 2007), but is a part of the core transcriptional feedback loop suppressing its own transcription (and that of per) by interfering with the actions of the CLK-BMAL1 heterodimer (Kume, et al. 1999,Jin, et al. 1999). Mammals also lack a homolog for timeless, possessing only a homolog of the Drosophila gene timeout (Benna, et al. 2000), a gene whose potential role in the clock is less clear and less crucial than that of timeless (Gustafson and Partch, 2015,Benna, et al. 2010).

The Lepidoptera harbour both types of cryptochrome (Drosophila-like cry1 and mammal-like cry2) (Tomioka and Matsumoto, 2010), as well as homologs of timeless and timeout (Tomioka and Matsumoto, 2015). The two cryptochromes have been shown to act in a similar way to their Drosophila and mammal counterparts; cry1 functions as a light receptor and cry2 serves as a transcriptional repressor (Zhu, et al. 2008). Of the major insect orders, the Hymenoptera arguably possess the most mammalian-like core clock architecture, possessing cry2 and timeout but neither 
74 cry1 nor timeless (Tomioka and Matsumoto, 2015,Yuan, et al. 2007). In addition

75 to these molecular similarities, there is evidence that the transcriptional profiles

76 of these genes match more closely the mammalian model than the Drosophila

77 model (Rubin, et al. 2006). Light-entrained circadian rhythms have been

78 demonstrated in the Hymenoptera, but the question of light detection in the

79 Hymenopteran clock remains an open one.

80 Nasonia vitripennis is a parasitoid wasp, which as a research model offers

81 advantages over other hymenopterans, including a fully sequenced genome

82 (Werren, et al. 2010), systemic RNAi (Lynch and Desplan, 2006), a robust and

83 well-characterised circadian response (Bertossa, et al. 2013), a fully functional

84 DNA methylation kit (Park, et al. 2011), and a history as a model for

85 photoperiodism (Saunders, 1969).

86 In this study, we advance Nasonia as an alternative circadian model by

87 using RNA-seq to profile whole-transcriptome gene expression in the Nasonia

88 head. As the Nasonia clock free-runs in both constant darkness and constant light

89 (Figure 1), we profiled both of these conditions to examine how the two

90 circadian transcriptomes differ. To our knowledge, this is the first circadian

91 RNA-seq study performed in an insect other than Drosophila, and the first study

92 to profile the circadian transcriptome oscillating under constant light.

93 Results

\section{$94 \quad$ Identifying rhythmic transcription}

95 We first performed an unbiased clustering analysis to ascertain the kinds of

96 expression patterns present in the data. To this end, Mfuzz (Kumar and E

97 Futschik, 2007) was used to carry soft c-means clustering, a method which is less 
sensitive to biological noise than traditional clustering (Futschik and Carlisle,

2005). After filtering (see Methods), thirty clusters were generated for each

100

101

102

103

104

105

106

107

108

109

110

111

112

113

114

115

116

117

118

119

120 121 at isolated time-points with no obvious specific shared function. This is in 122 contrast with results in Drosophila and mammals, where some core clock genes

and Westermark, 2014). At false discovery rate (FDR) threshold of 0.1 we identified 1,057 rhythmic transcripts in DD and 929 in LL (Table S1, S2).

Rhythmic transcripts $(\mathrm{q}<0.1)$ were sorted by phase, peak shape, and significance, and plotted (Figure 2A). Examining the phase distribution (Figure 2B), it is apparent that the majority of transcripts show peak expression early in the subjective morning/afternoon or in the subjective night, with fewer transcripts peaking at intermediate times. This disparity in phase is greater in the transcripts which show rhythmic expression in both DD and LL; less than $12 \%$ of transcripts in DD and less than 5\% in LL show peak expression at intermediate times (Figure $2 \mathrm{~B})$. The majority of these transcripts $(\sim 87 \%)$ exhibit a similar (+-4 hrs) phase in LL to their phase in DD.

Similarly to Drosophila (Hughes, et al. 2012) and mammals (Hughes, et al. 2009), the majority of transcripts show only small cyclic changes in expression amplitude over the day; over $80 \%$ of reliably quantified (see Methods) transcripts in both conditions have amplitudes (peak expression divided by trough expression) of 2 or less. In both DD and LL, transcripts with exceptionally high amplitudes ( $>4$ ) are transcripts with unusually low or high measurements 
123 exhibit very high amplitude oscillations (Hughes, et al. 2009,Hughes, et al.

124 2012,Li, et al. 2015).

125

\section{Canonical clock genes and comparison with Drosophila}

127 The canonical clock genes were examined for rhythmicity both at the transcript

128 level and via an additional RAIN analysis at the gene level. The q-values (FDR

129 adjusted p-values) for the canonical clock genes are shown in supplementary

130 table S3. We found a rather limited evidence for rhythmicity in these genes

131 which included $p d p 1 e(\mathrm{q} \sim 0.1$, LL and DD), shaggy $(\mathrm{q}<0.1, \mathrm{DD})$, and Clok $(\mathrm{q} \sim$

$1320.1, \mathrm{LL})$. At a less stringent FDR ( $\mathrm{q}<0.2)$, per, cyc, Dbt and $c w o$ were rhythmic in

133 DD, while cry and $c y c$, were oscillating in LL. For comparison between splice

134 variants and conditions, median expression levels of the canonical clock genes

135 and their transcripts for both DD and LL are shown in supplementary table S4.

136 We compared the transcripts identified as cycling in Nasonia heads with

137 the transcripts identified as cycling in Drosophila heads. For these purposes, we

138 used a list of genes identified in a meta-analysis study of Drosophila circadian

139 microarray data as being rhythmically expressed in either LD or DD (Keegan, et

140 al. 2007). Of 173 genes identified as rhythmic in Drosophila, 33 genes

141 (Supplementary table S5) were found to also be rhythmic in Nasonia (either in

142 LL or DD, $q<0.1)$, no more than would be expected by chance $(p=0.11$,

143 hypergeometric test).

144

145 Functions of rhythmic genes

146 To capture the general functions that rhythmic genes may fulfil in Nasonia, we

147 tested a broader set of rhythmic genes (FDR $<0.2$ in RAIN) for GO term 
148 overrepresentation (Davies and Tauber, 2015a), revealing 94 GO terms

149 overrepresented for genes rhythmic in DD (including 'response to light stimulus',

150 'proteasome complex', and 'generation of neurons', Supplementary table S6) and

151123 terms for genes rhythmic in LL (including 'locomotion','proteasome

152 complex', and 'response to external stimulus', Supplementary table S7), 25 of

153 which were shared between both conditions (Figure 3). Shared terms include

154 terms related to neurons, signal transmission, and responses to stimuli. Notably,

155 all four Nasonia opsins were found to exhibit similar transcriptional profiles in

156 LL and DD, with low expression in the morning and high expression in the

157 evening.

158 It has previously been demonstrated that the timing of different (or indeed

159 opposing) biological processes can be controlled through the circadian

160 regulation of groups of genes (Sancar, et al. 2015,Zhang, et al. 2014).

161 Unsupervised clustering methods have previously been established as a useful

162 method for functional characterisation of circadian genes (Nguyen, et al. 2014).

163 To establish whether temporal separation of functions occurs in Nasonia, we

164 therefore returned to the expression clustering analysis. Firstly, we employed

165 hypergeometric tests to identify clusters with an overrepresentation of rhythmic

166 genes (Figure 4, Supplementary table S8 and S9). Clusters which were found to

167 have a significant rhythmic component $(\mathrm{q}<0.05$, supplementary tables $\mathrm{S} 8$ and

168 S9) were analysed for overrepresented G0 terms. Examples of clusters with

169 enriched functions include clusters DD7 and LL20 which are significantly

170 enriched for catalytic activity GO terms, especially genes involved in the

171 proteasome, and clusters DD24 and LL6 which are both involved in circadian

172 and neural processes. Other clusters (DD1 and DD2) did not turn up any 
173 overrepresented GO terms and are thus likely comprised of genes with a wide 174 range of functions.

175

176 Transcriptional differences between constant darkness and constant light

177 To examine whether differences in circadian period seen in locomotor activity

178 between DD and LL could also be detected in transcriptional rhythms, we fitted

179 parametric models with a range of periods to transcripts rhythmic in both

180 conditions $(\mathrm{q}<0.1)$. For those transcripts with statistically significant fits to the

181 model in both conditions ( $q<0.1$, see Methods), we took the period with the best

182 fit and compared these periods between conditions. Overall, transcripts in LL

183 showed a significantly ( $p<3.9 \mathrm{e}-09$, Wilcoxon rank sum test) shorter (median

184 24) period than those in DD (median 25.4), mirroring the behavioural

185 differences in period.

186 We have also tested for differential expression between DD and LL. In the

187 absence of biological replicates, we analysed differential expression using a fold-

188 change approach. We used 1.5 fold change as a cut-off for differential expression

189 (Dalman, et al. 2012), yielding 1,488 genes expressed higher in DD than LL and

190971 genes expressed higher in LL than DD (Figure 5). Genes more highly

191 expressed in DD were significantly enriched $(q<0.01)$ for genes involved in

192 various forms of catalytic activity (Supplementary table S10), including the vast

193 majority of proteasome genes (>75\%). Genes more highly expressed in LL were

194 enriched for a small number of terms including 'plasmalemma' and 'sequence-

195 specific DNA binding' (Supplementary table S11). 
197 This study provides the first insights into global transcriptional oscillation in

198 Nasonia. With RNA-seq, we profiled the circadian transcription of $>26,000$

199 transcripts in Nasonia in either DD or LL. At a relatively stringent FDR $(\mathrm{q}<0.1)$,

200 we identified 1,057 cycling transcripts in DD and 929 cycling transcripts in LL.

201 These transcripts correspond to a cycling fraction of $6.7 \%$ and $5.9 \%$ of all

202 transcripts analysed in DD and LL respectively. These figures are comparable to

203 cycling fractions reported in various organisms and tissues, generally between

$2042 \%$ and $10 \%$ of the transcriptome (Michael and McClung, 2003).

205 In both conditions, cycling transcripts were found to cycle at low

206 amplitudes (mostly $<2$ fold) and with a limited, bimodal, range of phases. This is

207 in contrast to microarray/RNA-seq studies in Drosophila, where transcripts were

208 found to cycle with a broader range of phases (Rodriguez, et al. 2013) and

209 studies in both mammals and Drosophila, which have identified a group of high-

210 amplitude (> 4-fold) cycling genes among the low-amplitude majority (Akhtar, et

211 al. 2002). High amplitude cyclers typically include clock genes (Akhtar, et al.

212 2002,Hughes, et al. 2012). The low oscillations of the Nasonia head

213 transcriptome render the expression profiles of the canonical clock genes

214 difficult to resolve (Covington, et al. 2008). This issue may also contribute to the

215 discordance between the various circadian microarray studies in Drosophila

216 (Keegan, et al. 2007).

217 An emerging property of the circadian transcriptome in Nasonia is the

218 temporal separation of function by phase (Fig 2). Notably, genes involved in

219 catalytic activity were strongly overrepresented in morning-peaking transcripts.

220 This is in line with other studies which show catalytic activity confined to the 
221 morning in fungi (Sancar, et al. 2015), in agreement with a general observation

222 that an important (or even primary) function of circadian clocks (Hurley, et al.

2232015 ) is to temporally separate catabolism and anabolism. Although we did not

224 detect an overrepresentation of anabolic genes within the cyclic transcripts,

225 expression clusters DD10 and LL24 (Supplementary figures S1 and S2) did show

226 strong overrepresentation (Supplementary tables S12 and S13) for genes

227 involved in cytosolic ribosomal genes $(q<3 . e-56)$ and cellular anabolism $(q<2 e-$

228 06). These clusters exhibit an antagonistic expression pattern to the expression

229 clusters containing the catabolic genes, suggesting that catabolism and

230 anabolism are indeed separated by the circadian clock in Nasonia.

231 The comparison of expression between LL and DD reveals that a majority

232 of genes involved in the proteasome and a broader set of genes involved in

233 catabolism, are more highly expressed in DD than LL. As turnover rates of clock

234 proteins have shown to be coupled with changes in the circadian period (Syed, et

235 al. 2011,He and Liu, 2005), up-regulation of the proteasome may provide an

236 explanation for differences in period observed between DD and LL.

237 Although the genes which cycle in Drosophila largely differ from those

238 cycling in Nasonia, the functions fulfilled by CCGs in Nasonia are similar to the

239 functions filled by CCGs in Drosophila. Examples of functions shared by CCGs in

240 the Drosophila and Nasonia heads are: various aspects of metabolism (Rodriguez,

241 et al. 2013,Ueda, et al. 2002,Ceriani, et al. 2002,Claridge-Chang, et al. 2001),

242 phototransduction (Ueda, et al. 2002,Rodriguez, et al. 2013), synaptic/nervous

243 functions (McDonald and Rosbash, 2001b,Ceriani, et al. 2002,Claridge-Chang, et

244 al. 2001), oxidoreductase activity (Claridge-Chang, et al. 2001), mating behaviour 
245 (Rodriguez, et al. 2013), and immunity (McDonald and Rosbash, 2001b,Ceriani, 246 et al. 2002).

247 We identified cycling of genes involved in response to light, particularly

248 all four Nasonia opsins. These opsins, along with associated gPCRs, cycle with a

249 similar phase and are all more highly expressed in LL than in DD (Supplementary

250 figure S6). Daily and circadian changes in opsin expression have been

251 demonstrated in other organisms (e.g. mice (Bowes, et al. 1988), zebrafish (Li, et

252 al. 2005), honeybee (Sasagawa, et al. 2003)), and opsin expression is generally

253 found to be up-regulated in response to light (Yan, et al. 2014). Characterising

254 the opsins in Nasonia is likely to provide insights into the light input pathway

255 into the clock, particularly as Nasonia does not possess other obvious light input

256 candidate genes such as Drosophila-like CRY1 (Bertossa, et al. 2014) or Pteropsin

257 (Velarde, et al. 2005) (Supplementary figure S6).

\section{Data availability}

259 We have made the expression profile for each transcript in both conditions

260 available on WaspAtlas (Davies and Tauber, 2015b). Data have been archived in

261 the NCBI short read archive (SRA), with accession number PRJNA318159.

\section{Methods}

\section{Maintenance and sample collection}

264 Stocks of Nasonia vitripennis (strain AsymCX) were maintained at $25^{\circ} \mathrm{C}$ on

265 blowfly pupal hosts in 12:12 light:dark cycles. To obtain male wasps for

266 experiments, groups of eight females were isolated at the yellow pupal stage and

267 transferred onto fresh hosts upon eclosion. The resulting male progeny were

268 collected upon eclosion and moved onto vials with a 30\% sucrose agar medium, 
269 in groups of 20. During entrainment (four full days in an LD 12:12 cycle) and

270 collection, wasps were kept in four light boxes in the same incubator at $19^{\circ} \mathrm{C}$.

271 Starting at CT1, wasps were collected every four hours and snap-frozen in liquid

272 nitrogen and immediately transferred to $-80^{\circ} \mathrm{C}$. Wasps were collected

273 sequentially from light box to light box every four hours to minimise disturbance

274 of wasps, and so that wasps were collected from each light box once every 16

275 hours, thereby minimising the effect of variations within light boxes.

276 Temperature and light recordings were taken during the experiment, and can be

277 viewed in Supplementary file S2. To verify that wasps entrained correctly to the

278 experimental conditions and that free-running behaviour was as expected,

279 individual male wasps were isolated and locomotor activity was monitored.

280 Behavioural recordings of individual male wasps in experimental conditions can

281 be seen in Supplementary figure S7, ruling out behavioural differences caused by

282 inter light box variations in light intensity in LL, though not transcriptional

283 differences.

284

285 RNA extraction, sequencing, and read mapping

286 RNA was extracted from pooled groups of 50 heads for each sample, using Trizol

287 RNA extraction protocol, and followed by clean-up using the RNAeasy spin

288 column kit (Qiagen). Samples were polyA selected and sequenced at Glasgow

289 Polyomics (University of Glasgow, United Kingdom) on the Illumina NextSeq500

290 platform, resulting in approximately 20 million 75bp paired-end reads per

291 sample.

292 Read mapping was achieved with Tophat2 (v2.1.0)(Trapnell, et al. 2012)

293 against the Nasonia Nvit_2.1 NCBI annotation. As the purpose of this study was 
294 not to identify novel splice variants or improve on existing annotation, novel

295 junction detection was disabled for accurate quantification of known transcripts.

296 Mean mapping efficiency was above 90\% for both conditions (Supplementary

297 table S14). Read quantification was performing using the DEseq normalisation

298 method (Anders and Huber, 2010). All 24 samples from both conditions were

299 grouped together to allow comparison between as well as within conditions.

300

\section{Expression profile clustering}

302 Isoform expression profiles were first filtered to include only those isoforms

303 with no missing values at any time-point in either condition. Expression values

304 were standardised using the 'Standardise' function in Mfuzz (Kumar and E

305 Futschik, 2007). The ‘cselection' function in Mfuzz was used to select an

306 appropriate c-value for the c-means clustering (default parameters; $m=1.25$ ).

307 Based on this analysis, thirty fuzzy clusters were generated for each condition

308 using the fuzzification parameter $\mathrm{m}=1.25$.

309

310 Rhythmic expression analysis

311 RAIN (Thaben and Westermark, 2014) was used on all filtered isoforms (i.e.

312 those with no missing values at any timepoint) in either condition to detect

313 rhythmic isoforms at a period of 24 hours. As a non-parametric method, RAIN

314 only facilitates detection of rhythmic isoforms with periods which are a multiple

315 of the sample resolution (in this case $4 \mathrm{hr}$ ). The p-values produced by RAIN were

316 corrected to q-values using the Benjamini-Hochberg method (Benjamini and

317 Hochberg, 1995). This method was repeated using expression values for genes 
318 rather than transcripts for the clock gene analysis (i.e. the summed expression

319 values for all known transcripts of a particular gene).

320 Maximum fold changes in expression were calculated by normalising per-

321 condition expression values by the median value and calculating the ratio from

322 the lowest expression over 48 hours to the highest. Reliably quantified

323 transcripts are defined as those those transcripts where the absolute FPKM

324 value is 5 or above at all timepoints, the threshold for this set at a similar level to

325 other analyses (Hughes, et al. 2012).

326 To analyse the period of rhythmic transcripts, we fitted parametric

327 waveforms with a variety of periods ( 20 to $28 \mathrm{hrs}$ in steps of $0.2 \mathrm{hrs}$ ) to all

328 transcripts identified as rhythmic $(\mathrm{q}<0.1)$ in both conditions. This FDR

329 threshold is in line with, or more strict, than thresholds chosen in other similar

330 studies (Hughes, et al. 2012,Huang, et al. 2013,Keegan, et al. 2007). Those

331 transcripts (85 in total) which showed a significant $(q<0.1)$ fit to the model in

332 both conditions were analysed in terms of their best fitting period.

333 GO term overrepresentation was performed in WaspAtlas (Davies and

334 Tauber, 2015b) using the Nvit_2.1 NCBI annotation dataset. All hypergeometric

335 tests were performed within R using the 'phyper' function. Clusters with

336 rhythmic components were identified by collapsing the fuzzy clusters into hard

337 clusters using the 'cluster' property of the Mfuzz object, performing

338 hypergeometric tests to identify clusters with enrichment for rhythmic

339 transcripts. Thirty tests were performed for each condition (i.e. for all clusters),

340 and were corrected per-condition using the Benjamini-Hochberg method in R (R

341 Development Core Team, 2008). 
343 melanogaster were obtained from a meta-study of circadian microarray data

344 (Keegan, et al. 2007). The 214 obtained FlyBase identifiers were converted to the

345 latest identifiers using the validation tool, resulting in 218 unique identifiers (the

346 increase in identifiers can be attributed to previous identifiers referring to

347 multiple genes in the current annotation). Orthologs for these Drosophila genes

348 were obtained through WaspAtlas, retrieving orthologs for 135 genes which

349 mapped to 173 unique Nasonia genes due to gene duplications, etc. This set of

350173 genes was compared with the number of genes with rhythmic transcripts

351 that would be expected by chance using a hypergeometric test.

\section{Phylogenetic analysis of opsin genes}

354 Opsin genes were searched for using NCBI BLASTP using six species; Apis

355 mellifera, Bombyx mori, Drosophila melanogaster, Mus musculus, Nasonia

356 vitripennis, and Homo sapiens, using the Nasonia Lop1 protein sequence as a

357 query. BLAST results were inspected and 7e-19 was chosen as an appropriate

358 cut-off to include all opsin sequences. Sequences were aligned by ClustalW in

359 MEGA (Tamura, et al. 2007) and a maximum likelihood tree generated using

360 default parameters. Duplicated sequences were manually removed, and

361 sequences renamed for display on the tree. Full protein name to shortened

362 display name translations can be found in supplementary table S15. 
364 References

365 Akhtar RA, Reddy AB, Maywood ES, Clayton JD, King VM, Smith AG, Gant TW,

366 Hastings MH, Kyriacou CP. 2002. Circadian cycling of the mouse liver

367 transcriptome, as revealed by cDNA microarray, is driven by the

368 Suprachiasmatic Nucleus. Current Biology, 12: 540-550.

369 Anders S and Huber W. 2010. Differential expression analysis for sequence count 370 data. Genome Biol. 11: R106-2010-11-10-r106. Epub 2010 Oct 27.

371 Benjamini Y and Hochberg Y. 1995. Controlling the False Discovery Rate: A 372 Practical and Powerful Approach to Multiple Testing. Journal of the Royal

373 Statistical Society.Series B (Methodological) 57: 289-300.

374 Benna C, Bonaccorsi S, Wulbeck C, Helfrich-Forster C, Gatti M, Kyriacou CP, Costa

375 R, Sandrelli F. 2010. Drosophila timeless2 is required for chromosome stability

376 and circadian photoreception. Curr. Biol. 20: 346-352.

377 Benna C, Scannapieco P, Piccin A, Sandrelli F, Zordan M, Rosato E, Kyriacou CP,

378 Valle G, Costa R. 2000. A second timeless gene in Drosophila shares greater

379 sequence similarity with mammalian tim. Curr. Biol. 10: R512-3.

380 Bertossa RC, van de Zande L, Beukeboom LW, Beersma DG. 2014. Phylogeny and

381 oscillating expression of period and cryptochrome in short and long

382 photoperiods suggest a conserved function in Nasonia vitripennis. Chronobiol.

383 Int. 31: 749-760. 
384 Bertossa RC, van Dijk J, Diao W, Saunders D, Beukeboom LW, Beersma DG. 2013.

385 Circadian rhythms differ between sexes and closely related species of Nasonia

386 wasps. PLoS One 8: e60167.

387 Bowes C, van Veen T, Farber DB. 1988. Opsin, G-protein and 48-kDa protein in

388 normal and rd mouse retinas: developmental expression of mRNAs and proteins

389 and light/dark cycling of mRNAs. Exp. Eye Res. 47: 369-390.

390 Busza A, Emery-Le M, Rosbash M, Emery P. 2004. Roles of the two Drosophila

391 CRYPTOCHROME structural domains in circadian photoreception. Science 304:

392 1503-6.

393 Ceriani MF, Darlington TK, Staknis D, Mas P, Petti AA, Weitz CJ, Kay SA. 1999.

394 Light-dependent sequestration of TIMELESS by CRYPTOCHROME. Science 285:

395 553-6.

396 Ceriani MF, Hogenesch JB, Yanovsky M, Panda S, Straume M, Kay SA. 2002.

397 Genome-Wide Expression Analysis in Drosophila Reveals Genes Controlling

398 Circadian Behavior. J. Neurosci. 22: 9305-9319.

399 Claridge-Chang A, Wijnen H, Naef F, Boothroyd C, Rajewsky N, Young MW. 2001.

400 Circadian regulation of gene expression systems in the Drosophila head. Neuron

$401 \quad 32: 657-671$.

402 Covington MF, Maloof JN, Straume M, Kay SA, Harmer SL. 2008. Global

403 transcriptome analysis reveals circadian regulation of key pathways in plant

404 growth and development. Genome Biol. 9: R130. 
405 Dalman MR, Deeter A, Nimishakavi G, Duan ZH. 2012. Fold change and p-value

406 cutoffs significantly alter microarray interpretations. BMC Bioinformatics $\mathbf{1 3}$

407 Suppl 2: S11-2105-13-S2-S11.

408 Davies NJ and Tauber E. 2015a. WaspAtlas: a Nasonia vitripennis gene database

409 and analysis platform. Database (Oxford) 2015: bav103. Print 2015.

410 Davies NJ and Tauber E. 2015b. WaspAtlas: a Nasonia vitripennis gene database

411 and analysis platform. Database (Oxford) 2015: 10.1093/database/bav103. Print 4122015.

413 Futschik ME and Carlisle B. 2005. Noise-robust soft clustering of gene expression 414 time-course data. J. Bioinform Comput. Biol. 3: 965-988.

415 Grima B, Lamouroux A, Chelot E, Papin C, Limbourg-Bouchon B, Rouyer F. 2002.

416 The F-box protein slimb controls the levels of clock proteins period and timeless.

417 Nature 420: 178-82.

418 Gustafson CL and Partch CL. 2015. Emerging models for the molecular basis of 419 mammalian circadian timing. Biochemistry 54: 134-149.

420 He Q and Liu Y. 2005. Degradation of the Neurospora circadian clock protein 421 FREQUENCY through the ubiquitin-proteasome pathway. Biochem. Soc. Trans. 422 33: 953-956.

423 Huang W, Ramsey KM, Marcheva B, Bass J. 2011. Circadian rhythms, sleep, and 424 metabolism. J. Clin. Invest. 121: 2133-2141. 
425 Huang Y, Ainsley JA, Reijmers LG, Jackson FR. 2013. Translational profiling of

426 clock cells reveals circadianly synchronized protein synthesis. PLoS Biol. 11:

$427 \mathrm{e} 1001703$.

428 Hughes ME, DiTacchio L, Hayes KR, Vollmers C, Pulivarthy S, Baggs JE, Panda S,

429 Hogenesch JB. 2009. Harmonics of circadian gene transcription in mammals.

$430 \quad$ PLoS Genet 5: e1000442.

431 Hughes ME, Grant GR, Paquin C, Qian J, Nitabach MN. 2012. Deep sequencing the

432 circadian and diurnal transcriptome of Drosophila brain. Genome Res. 22: 1266-

4331281.

434 Hurley JM, Loros JJ, Dunlap JC. 2015. The circadian system as an organizer of 435 metabolism. Fungal Genet. Biol.

436 Jin X, Shearman LP, Weaver DR, Zylka MJ, de Vries GJ, Reppert SM. 1999. A

437 molecular mechanism regulating rhythmic output from the suprachiasmatic

438 circadian clock. Cell 96: 57-68.

439 Keegan KP, Pradhan S, Wang JP, Allada R. 2007. Meta-analysis of Drosophila

440 circadian microarray studies identifies a novel set of rhythmically expressed

441 genes. PLoS Comput. Biol. 3: e208.

442 Kelleher FC, Rao A, Maguire A. 2014. Circadian molecular clocks and cancer.

443 Cancer Lett. 342: 9-18.

444 Ko HW, Jiang J, Edery I. 2002. Role for Slimb in the degradation of Drosophila

445 Period protein phosphorylated by Doubletime. Nature 420: 673-678. 
446 Konopka R and Benzer S. 1971. Clock mutants of Drosophila melanogaster. Proc.

447 Natl. Acad. Sci. USA 68: 2112-2116.

448 Kumar L and E Futschik M. 2007. Mfuzz: a software package for soft clustering of 449 microarray data. Bioinformation 2: 5-7.

450 Kume K, Zylka MJ, Sriram S, Shearman LP, Weaver DR, Jin X, Maywood ES,

451 Hastings MH, Reppert SM. 1999. mCRY1 and mCRY2 are essential components of 452 the negative limb of the circadian clock feedback loop. Cell 98: 193-205.

453 Li, J., Grant, G. R., Hogenesch, J. B., and Hughes, M. E. 2015. Chapter Sixteen 454 Considerations for RNA-seq Analysis of Circadian Rhythms. In Methods in 455 Enzymology (ed. Amita Sehgal), pp. 349-367. Academic Press, .

456 Li P, Temple S, Gao Y, Haimberger TJ, Hawryshyn CW, Li L. 2005. Circadian

457 rhythms of behavioral cone sensitivity and long wavelength opsin mRNA 458 expression: a correlation study in zebrafish. J. Exp. Biol. 208: 497-504.

459 Lynch JA and Desplan C. 2006. A method for parental RNA interference in the 460 wasp Nasonia vitripennis. Nat. Protoc. 1: 486-494.

461 Maury E, Ramsey KM, Bass J. 2010. Circadian Rhythms and Metabolic Syndrome:

462 From Experimental Genetics to Human Disease. Circulation Research 106: 447463462.

464 McDonald MJ and Rosbash M. 2001a. Microarray analysis and organization of 465 circadian gene expression in Drosophila. Cell 107: 567-578. 
466 McDonald MJ and Rosbash M. 2001b. Microarray analysis and organization of

467 circadian gene expression in Drosophila. Cell 107: 567-578.

468 Michael TP and McClung CR. 2003. Enhancer trapping reveals widespread

469 circadian clock transcriptional control in Arabidopsis. Plant Physiol. 132: 629-

470639.

471 Musiek ES, Xiong DD, Holtzman DM. 2015. Sleep, circadian rhythms, and the 472 pathogenesis of Alzheimer disease. Exp. Mol. Med. 47: e148.

473 Nguyen TT, Mattick JS, Yang Q, Orman MA, Ierapetritou MG, Berthiaume F, 474 Androulakis IP. 2014. Bioinformatics analysis of transcriptional regulation of 475 circadian genes in rat liver. BMC Bioinformatics 15: 83-2105-15-83.

476 Park J, Peng Z, Zeng J, Elango N, Park T, Wheeler D, Werren JH, Yi SV. 2011.

477 Comparative analyses of DNA methylation and sequence evolution using 478 Nasonia genomes. Mol. Biol. Evol. 28: 3345-3354.

479 Quera Salva MA, Hartley S, Barbot F, Alvarez JC, Lofaso F, Guilleminault C. 2011. 480 Circadian rhythms, melatonin and depression. Curr. Pharm. Des. 17: 1459-1470.

481 R Development Core Team. 2008. R: A Language and Environment for Statistical 482 Computing. R Foundation for Statistical Computing, Vienna, Austria.

483 Rodriguez J, Tang CH, Khodor YL, Vodala S, Menet JS, Rosbash M. 2013. Nascent484 Seq analysis of Drosophila cycling gene expression. Proc. Natl. Acad. Sci. U. S. A. 485 110: E275-84. 
486 Rosato E, Tauber E, Kyriacou CP. 2006. Molecular genetics of the fruit-fly

487 circadian clock. Eur. J. Hum. Genet. 14: 729-38.

488 Rubin EB, Shemesh Y, Cohen M, Elgavish S, Robertson HM, Bloch G. 2006.

489 Molecular and phylogenetic analyses reveal mammalian-like clockwork in the

490 honey bee (Apis mellifera) and shed new light on the molecular evolution of the

491 circadian clock. Genome Res. 16: 1352-1365.

492 Sancar C, Sancar G, Ha N, Cesbron F, Brunner M. 2015. Dawn- and dusk-phased

493 circadian transcription rhythms coordinate anabolic and catabolic functions in

494 Neurospora. BMC Biol. 13: 17-015-0126-4.

495 Sasagawa H, Narita R, Kitagawa Y, Kadowaki T. 2003. The expression of genes

496 encoding visual components is regulated by a circadian clock, light environment

497 and age in the honeybee (Apis mellifera). Eur. J. Neurosci. 17: 963-970.

498 Saunders DS. 1969. Diapause and photoperiodism in the parasitic wasp Nasonia

499 vitripennis, with special reference to the nature of the photoperiodic clock. Symp.

$500 \quad$ Soc. Exp. Biol. 23: 301-29.

501 Schaffer R, Landgraf J, Accerbi M, Simon V, Larson M, Wisman E. 2001.

502 Microarray analysis of diurnal and circadian-regulated genes in Arabidopsis.

$503 \quad$ Plant Cell 13: 113-123.

504 Scheiermann C, Kunisaki Y, Frenette PS. 2013. Circadian control of the immune

505 system. Nat. Rev. Immunol. 13: 190-198. 
506 Sehgal A, Price J, Man B, Young M. 1994. Loss of circadian behavioral rhythms

507 and per RNA oscillations in the Drosophila mutant timeless. Science 263: 1603-

5081606.

509 Syed S, Saez L, Young MW. 2011. Kinetics of doubletime kinase-dependent

510 degradation of the Drosophila period protein. J. Biol. Chem. 286: 27654-27662.

511 Takeda N and Maemura K. 2011. Circadian clock and cardiovascular disease. J.

512 Cardiol. 57: 249-256.

513 Tamura K, Dudley J, Nei M, Kumar S. 2007. MEGA4: Molecular Evolutionary

514 Genetics Analysis (MEGA) software version 4.0. Mol. Biol. Evol. 24: 1596-1599.

515 Thaben PF and Westermark P0. 2014. Detecting rhythms in time series with

516 RAIN. J. Biol. Rhythms 29: 391-400.

517 Tomioka K and Matsumoto A. 2010. A comparative view of insect circadian clock

518 systems. Cell Mol. Life Sci. 67: 1397-1406.

519 Tomioka K and Matsumoto A. 2015. Circadian molecular clockworks in non-

520 model insects. Current Opinion in Insect Science; Insect genomics * Development

521 and regulation 7: 58-64.

522 Trapnell C, Roberts A, Goff L, Pertea G, Kim D, Kelley DR, Pimentel H, Salzberg SL,

523 Rinn JL, Pachter L. 2012. Differential gene and transcript expression analysis of

524 RNA-seq experiments with TopHat and Cufflinks. Nat. Protoc. 7: 562-578. 
525 Ueda HR, Matsumoto A, Kawamura M, Iino M, Tanimura T, Hashimoto S. 2002.

526 Genome-wide Transcriptional Orchestration of Circadian Rhythms in Drosophila.

527 J. Biol. Chem. 277: 14048-14052.

528 Velarde RA, Sauer CD, O. Walden KK, Fahrbach SE, Robertson HM. 2005.

529 Pteropsin: A vertebrate-like non-visual opsin expressed in the honey bee brain.

$530 \quad$ Insect Biochem. Mol. Biol. 35: 1367-1377.

531 Werren JH, Richards S, Desjardins CA, Niehuis O, Gadau J, Colbourne JK, Nasonia

532 Genome Working Group, Werren JH, Richards S, Desjardins CA, et al. 2010.

533 Functional and evolutionary insights from the genomes of three parasitoid

$534 \quad$ Nasonia species. Science 327: 343-348.

535 Woelfle MA and Johnson CH. 2006. No promoter left behind: global circadian

536 gene expression in cyanobacteria. J. Biol. Rhythms 21: 419-431.

537 Yan S, Zhu J, Zhu W, Zhang X, Li Z, Liu X, Zhang Q. 2014. The expression of three

538 opsin genes from the compound eye of Helicoverpa armigera (Lepidoptera:

539 Noctuidae) is regulated by a circadian clock, light conditions and nutritional

$540 \quad$ status. PLoS One 9: e111683.

541 Yu W and Hardin PE. 2006. Circadian oscillators of Drosophila and mammals. J.

542 Cell. Sci. 119: 4793-4795.

543 Yuan Q, Metterville D, Briscoe AD, Reppert SM. 2007. Insect Cryptochromes:

544 Gene Duplication and Loss Define Diverse Ways to Construct Insect Circadian

545 Clocks. Mol. Biol. Evol. msm011. 
546 Zhang R, Lahens NF, Ballance HI, Hughes ME, Hogenesch JB. 2014. A circadian

547 gene expression atlas in mammals: implications for biology and medicine. Proc.

548 Natl. Acad. Sci. U. S. A. 111: 16219-16224.

549 Zhu H, Sauman I, Yuan Q, Casselman A, Emery-Le M, Emery P, Reppert SM. 2008.

550 Cryptochromes define a novel circadian clock mechanism in monarch butterflies

551 that may underlie sun compass navigation. PLoS Biol. 6: e4.

552

553 
Figure legend

Figure 1. Free-run behavioural rhythm in Nasonia. Representative actograms of individual Nasonia males in DD (left) and LL (right). Activity counts were sorted into 30 minute bins and plotted in blue. Yellow and grey backgrounds indicate lights on and lights off respectively. Gray and black bars below the actogram indicate the $12 \mathrm{hr}$ subjective day and night.

Figure 2. Circadian transcriptional rhythms. (A) Heatmap of median-normalised expression of rhythmic $(\mathrm{q}<0.1)$ transcripts in both constant darkness and constant light. (B) Histograms and heatmap of phases of rhythmic transcripts (q $<0.1$ in both conditions), showing bimodal phase distribution and overlap between the two conditions.

Figure 3. Enrichment of GO terms among cycling transcripts. (A) Bar plot of 10 top overrepresented GO terms (by gene proportion) for both DD and LL rhythmic genes. (B) Euler diagram showing the overlap of overrepresented terms in DD (blue) and LL (red).

Figure 4. Normalised expression of clusters with significant $(q<0.01)$ overrepresentations of rhythmic genes. Each transcript profile in each cluster is coloured by that gene's membership of the cluster.

Figure 5. Comparison of the DD and LL transcriptomes. (A) FPKM (log2) expression of transcripts in DD ( $\mathrm{x}$ axis) and LL (y axis), showing genes classified 
( $>1.5$ median fold change) as differentially expressed up in DD (blue) and up in LL (red). (B) Selected overrepresented ( $q<0.01)$ G0 terms for genes more highly expressed in DD. (C) Heatmap showing median-normalised expression for differentially expressed transcripts, in DD (left) and in LL (right), sorted by fold change. 


\section{Figure 1}
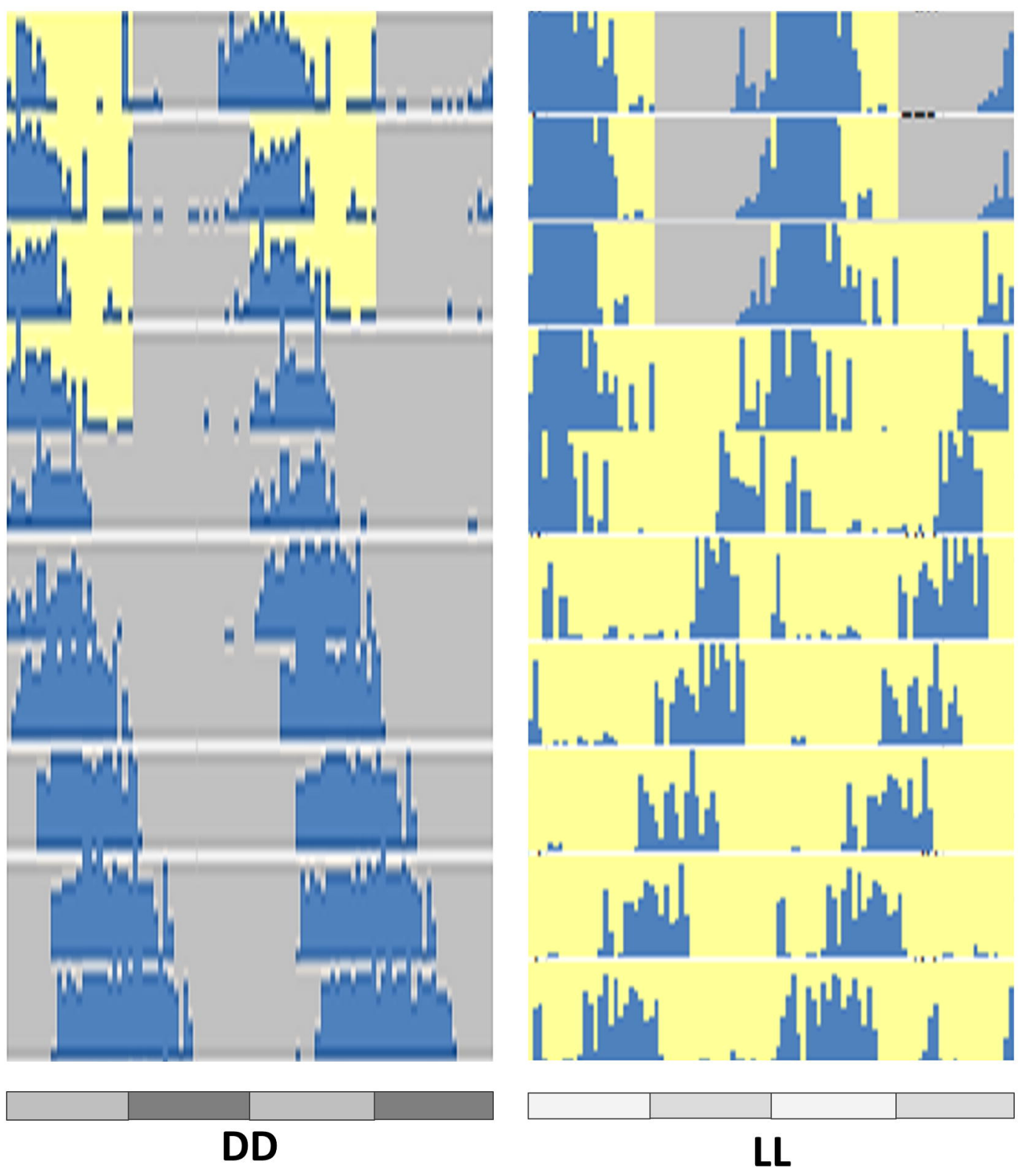


\section{Figure 3}

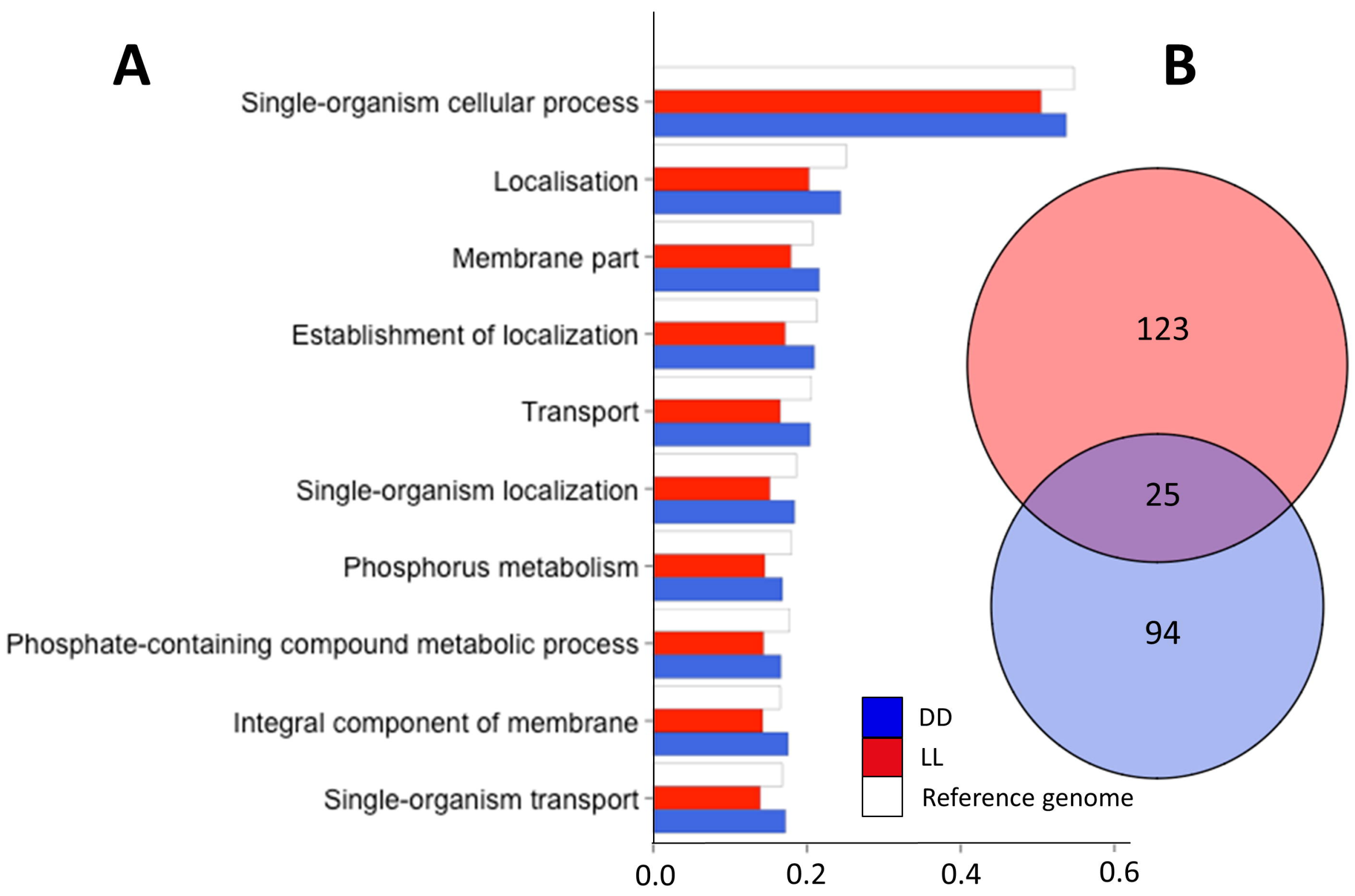


Figure 4

Cluster membership
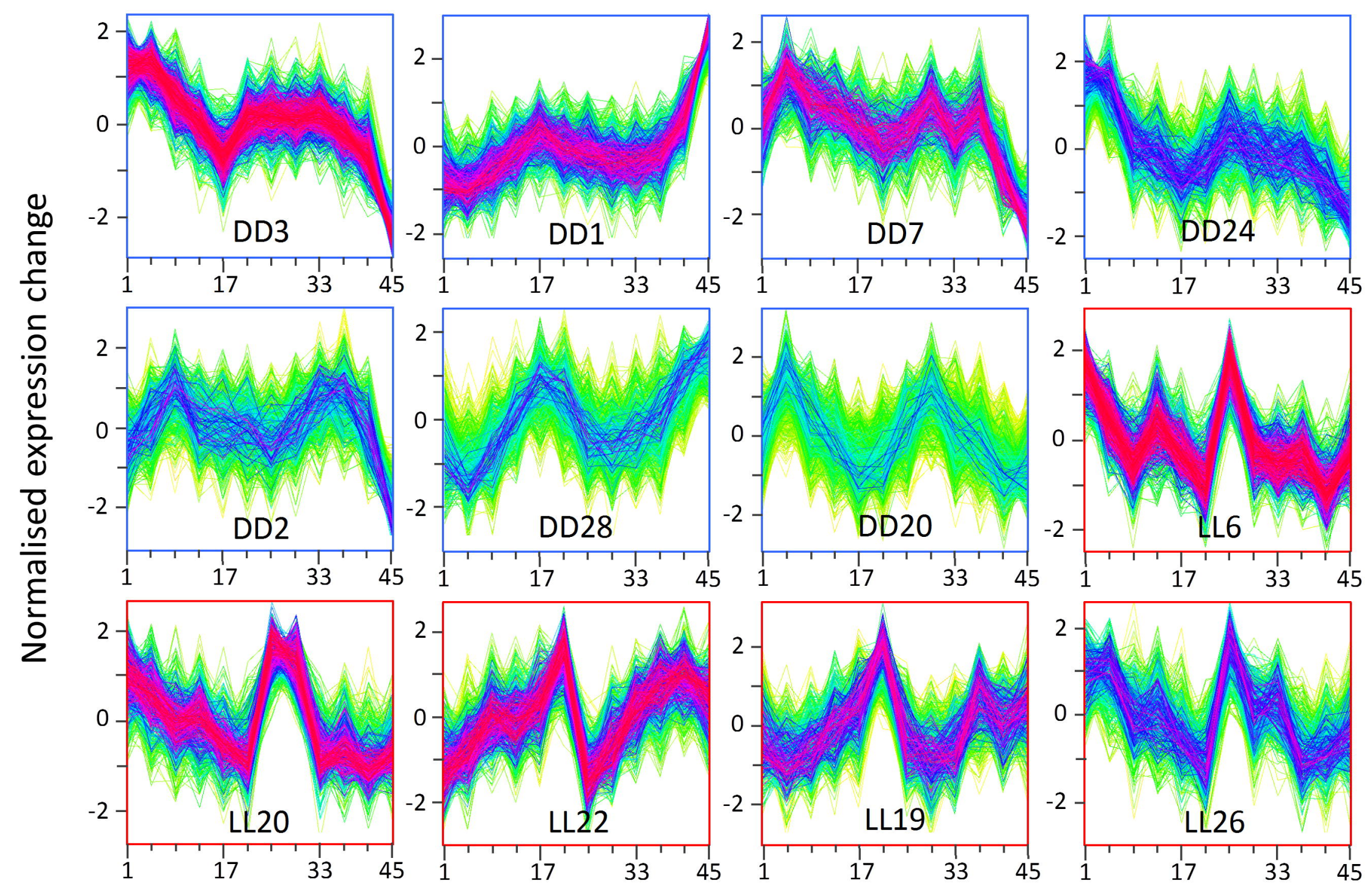

Time (CT) 
